\title{
CONCERNING COMPLETABLE MOORE SPACES ${ }^{1}$
}

\author{
G. M. REED
}

\begin{abstract}
The author obtains a generalization of well-known theorems due to Younglove and Fitzpatrick concerning the existence of dense metrizable subspaces in complete and completable Moore spaces. Based on this result, a new class of noncompletable Moore spaces is presented. In particular, an example of a separable noncompletable Moore space is given.
\end{abstract}

A (complete) Moore space is a space which satisfies Axiom 0 and has a (complete) development satisfying the first three parts (all) of Axiom 1 in [5]. The completely regular (complete) Moore spaces are precisely the (Čech complete) semistratifiable $p$-spaces [3]. A Moore space is completable provided that some complete Moore space contains it as a subspace. Each complete Moore space which is metrizable is completely metrizable [9]. However, there are examples of Moore spaces which are not completable ([10], [11], [7] and [6]).

A development $G=\left(G_{1}, G_{2}, \cdots\right)$ for the Moore space $S$ is said to satisfy Axiom $C$ at the point $p$ of $S$ if and only if, for each open set $D$ containing $p$, there is a positive integer $n$ such that each element of $G_{n}$ intersecting an element of $G_{n}$ which contains $p$ is contained in $D$. If $G$ is a development for the Moore space $S$, then $C(G)$, the set of all points at which $G$ satisfies Axiom C, is, if nonempty, a metrizable $G_{\delta}$-subset of $S$ ([4] and [12]).

In [12], Younglove proved that if $G$ is a development for the complete Moore space $S$ then $C(G)$ is dense in $S$. Fitzpatrick showed in [2] that a development $G$ for the completable Moore space $S$ need not satisfy Axiom $\mathrm{C}$ at any point of $S$ but that each completable Moore space does have a dense metrizable subspace.

The main result of this paper is Theorem 1: Each completable Moore space $S$ has a development $G$ such that $C(G)$ is dense in $S$. This improves Fitzpatrick's result in view of an example, given by the author in [8],

Presented to the Society, January 21,1971 ; received by the editors May 21, 1971.

AMS 1970 subject classifications. Primary 54E30, 54B05; Secondary 54G20.

Key words and phrases. Moore space, completable, metrizable, separable.

1 The work on this paper was done while the author was under the direction of Ben Fitzpatrick, Jr. The author would also like to acknowledge the many helpful suggestions made by the referee.

(c) American Mathematical Society 1973 
of a Moore space $S$ which has a dense metrizable subspace but for which there exists no development $G$ such that $C(G)$ is dense in $S$. Using Theorem 1 , the author is able to improve upon some other results concerning completable Moore spaces: (1) Theorem 2 establishes that each completable Moore space in which there does not exist an uncountable discrete collection of mutually exclusive open sets is separable. Armentrout had shown in [1] that each completable Moore space in which there does not exist an uncountable collection of mutually exclusive open sets is separable. (2) Theorem 3 establishes the existence of a separable noncompletable Moore space. Ott in [6] had obtained the same result under the assumption of the continuum hypothesis which is not required here.

Notation. If $H$ is a collection of point sets, then $H^{*}$ denotes the union of the elements of $H$.

THEOREM 1. Each completable Moore space $S$ has a development which satisfies Axiom $\mathrm{C}$ at each point of a dense subset of $S$.

Proof. Suppose $S$ is a subspace of the complete Moore space $Y$. Then $\bar{S}$, regarded as space, is a complete Moore space. Thus, consider the complete development $G_{1}, G_{2}, \cdots$ for $\bar{S}$.

For each positive integer $i$, let $H_{i}$ denote a maximal collection of mutually exclusive elements of $G_{i}$ such that $H_{i}^{*}$ is dense in $\bar{S}$. For each element $R$ in $H_{i}$ and each positive integer $j$, let $R_{j}=\left\{p \in R \mid\right.$ if $g \in G_{j}$ and $p \in g$, then $g$ is contained in $R\}$. Note that each $R_{j}$ is closed in $\bar{S}$ and $R=\bigcup_{j=1}^{\infty} R_{j}$. By Theorem 162 in [5], no open set in a complete Moore space is the union of countably many closed sets no one of which contains a nonempty open set. Thus for each $R$ in $H_{i}$, denote by $u_{R}$ a nonempty open set in $\bar{S}$ which is contained in $R_{m}$ for some positive integer $m$.

Now, if each of $i$ and $j$ is a positive integer, let $U_{i j}=\left\{u_{R} \mid R \in H_{i}\right.$ and $u_{R}$ is contained in $\left.R_{j}\right\}$. It follows that each $U_{i j}$ is a discrete collection of open sets in $\bar{S}$. Thus, for each pair of positive integers $i$ and $j$, denote by $K_{i j}$ a subset of $S$ which contains exactly one point of $u_{R} \cap S$ for each $u_{R}$ in $U_{i j}$. Consider $K=\bigcup_{i=1}^{\infty} \bigcup_{j=1}^{\infty} K_{i j}$. Since $K$ is the union of countably many point sets $K_{i j}$ such that each is covered by a discrete collection of open sets intersecting it at only one point, it follows from the proof of Theorem 5 in [2] that there exists a development $G^{\prime}$ for $\bar{S}$ which satisfies Axiom C at each point of $K$. It remains only to show that $K$ is dense in $S$.

To see that $K$ is dense in $\bar{S}$, consider the countable collection $\left\{H_{1}^{*}, H_{2}^{*}, \cdots\right\}$ of open sets each dense in the complete Moore space $\bar{S}$. By the proof of Theorem 164 in [5], it follows that $\bigcap_{i=1}^{\infty} H_{i}^{*}=M$ where $M$ is a dense subset of $\bar{S}$. But $M$ is contained in $\bar{K}$. For if $p \in M$ and $p$ is contained in the open set $D$, there exists a positive integer $n$ such that each element of $G_{n}$ containing $p$ is contained in $D$. Since $p \in H_{n}^{*}$, then 
$p \in R$ for some $R$ in $H_{n}$ and $u_{R}$, which contains a point of $K$, is contained in $D$. Thus $K$ is a subset of $S$ which is dense in $S$ and there exists a development $G^{\prime}$ for $S$ which satisfies Axiom $C$ at each point of $K$. It follows that $G_{1}^{\prime \prime}, G_{2}^{\prime \prime}, \cdots$, where for each $i, G_{i}^{\prime \prime}=\left\{g \cap S \mid g\right.$ in $\left.G_{i}^{\prime}\right\}$ is a development for $S$ which satisfies Axiom $\mathrm{C}$ at a dense subset of $S$.

In [8], under the assumption of the continuum hypothesis, the author gave an example of a Moore space in which there exists an uncountable collection of mutually exclusive open sets but in which there exists no such collection that is also discrete. Thus Theorem 2 is a generalization of Theorem 2.1 in [1].

THEOREM 2. Each completable Moore space $S$ in which there does not exist an uncountable discrete collection of mutually exclusive open sets is separable.

Proof. It follows from Theorem 1 that $S$ has a development $G$ such that $C(G)$ is dense in $S$. But in such a space, the existence of an uncountable collection of mutually exclusive open sets implies the existence of such a collection that is also discrete. For suppose that there exists an uncountable collection $H$ of mutually exclusive open sets in $S$. Let $M$ be a subset of $S$ containing one point of $h \cap C(G)$ for each $h$ in $H$. For each positive integer $i$, let $M_{i}=\left\{p \in M \mid\right.$ each element of $G_{i}$ intersecting an element of $G_{i}$ which contains $p$ is contained in the element of $H$ which contains $p\}$ and let $U_{i}=\left\{\operatorname{st}\left(p, G_{i}\right) \mid p \in M_{i}\right\}$. Note that for each $i, U_{i}$ is a discrete collection of mutually exclusive open sets. And since $M=\bigcup_{i=1}^{\infty} M_{i}$, there must exist a positive integer $k$ such that $U_{k}$ is uncountable.

Thus the completable Moore space $S$ satisfies the hypothesis of Theorem 2.1 in [1] and is therefore separable.

THEOREM 3. There exists a separable, noncompletable Moore space X.

Proof. In [8] the author gave an example of a Moore space $S$ for which there exists no development $G$ such that $C(G)$ is dense in $S$. Thus, by Theorem 1 , it suffices to show that there exists a separable Moore space $X$ which has $S$ as a subspace. Such a space $X$ will now be constructed by "sewing" onto $S$ countably many tangent disc spaces (i.e., Neimytzki planes).

1. Points of $S$. The points of $S$ are precisely all sequences $\left(p_{1}, p_{2}, \cdots\right.$, $\left.p_{k}, \cdots\right)$ of nonnegative real numbers such that $p_{k}>0, p_{i}$ is rational for $i<k$, and $p_{i}=0$ for $i>k$. For convenience we will express a point $p$ of $S$ as $p=\left(p_{1}, p_{2}, \cdots, p_{k}, 0, \cdots\right)$ where $k$ is the greatest integer such that $p_{k}$ is positive. 
2. Regions (basic open sets) of $S$. Suppose $n$ is a positive integer and $p=\left(p_{1}, p_{2}, \cdots, p_{k}, 0, \cdots\right)$ is a point of $S$. (i) If $p_{k}$ is irrational, then let

$$
\begin{gathered}
r_{1}^{n}(p)=\left\{\left(p_{1}, p_{2}, \cdots, p_{k-1}, t_{k}^{1}, t_{k+1}^{1}, 0, \cdots\right) \text { in } S \mid\right. \\
\left.0 \leqq t_{k+1}^{1} \leqq 1 / n, t_{k}^{1}=p_{k}+t_{k+1}^{1} \text { and } t_{k}^{1} \text { is rational }\right\} \\
r_{2}^{n}(p)=\left\{\left(p_{1}, p_{2}, \cdots, p_{k-1}, t_{k}^{1}, t_{k+1}^{2}, t_{k+2}^{2}, 0, \cdots\right) \text { in } S \mid\right. \text { there exists } \\
\left(p_{1}, p_{2}, \cdots, p_{k-1}, t_{k}^{1}, t_{k+1}^{1}, 0, \cdots\right) \text { in } r_{1}^{n}(p), 0 \leqq t_{k+2}^{2} \leqq 1 / n, \\
\left.t_{k+1}^{2}=t_{k+1}^{1}+t_{k+2}^{2}, \text { and } t_{k+1}^{2} \text { is rational }\right\}, \\
\cdot \\
\cdot \\
r_{i}^{n}(p)=\left\{\left(p_{1}, p_{2}, \cdots, p_{k-1}, t_{k}^{1}, t_{k+1}^{2}, \cdots, t_{k+i-1}^{i}, t_{k+i}^{i}, 0, \cdots\right) \text { in } S \mid\right. \text { there } \\
\text { exists }\left(p_{1}, p_{2}, \cdots, p_{k-1}, t_{k}^{1}, t_{k+1}^{2}, \cdots, t_{k+i-2}^{i-1}, t_{k+i-1}^{i-1}, 0, \cdots\right) \\
\text { in } r_{i-1}^{n}(p), 0 \leqq t_{k+i}^{i} \leqq 1 / n, t_{k+i-1}^{i}=t_{k+i-1}^{i-1}+t_{k+i}^{i}, \\
\text { and } \left.t_{k+i-1}^{i} \text { is rational }\right\},
\end{gathered}
$$

and continue this process to define $r_{j}^{n}(p)$ for each positive integer $j$. Let $g_{n}(p)=\bigcup_{j=1}^{\infty} r_{j}^{n}(p) \cup\{p\}$. (ii) If $p_{k}$ is rational, let $r_{1}^{n}(p)=\left\{\left(p_{1}, p_{2}, \cdots, p_{k}, \cdots, p_{m}, 0, \cdots\right)\right.$ in $S \mid m>k$,

and

$$
\left.0<p_{i} \leqq 1 / n \text { for } k<i \leqq m\right\}
$$

$r_{2}^{n}(p)=\bigcup\left\{g_{n}(q) \mid q=\left(q_{1}, q_{2}, \cdots, q_{m}, 0, \cdots\right)\right.$

in $r_{1}^{n}(p)$ and $q_{m}$ is irrational $\}$.

Let $g_{n}(p)=r_{1}^{n}(p) \cup r_{2}^{n}(p) \cup\{p\}$. Now, $g$ is a region for $S$ if and only if there exist a positive integer $n$ and a point $p$ of $S$ such that $g=g_{n}(p)$.

For each positive integer $i$, let $G_{i}^{\prime}=\left\{g_{i}(p) \mid p \in S\right\}$. For each positive integer $j$, let $G_{j}=\bigcup_{i=j}^{\infty} G_{i}^{\prime}$. It follows that $S$ is a Moore space and $G_{1}, G_{2}, \cdots$ is a development for $S$.

3. Construction of $X$. As noted in [8], $S$ is the sum of countably many "lines" where each line can be expressed as $\left\{\left(p_{1}, p_{2}, \cdots, p_{k-1}, y, 0, \cdots\right)\right.$ in $S \mid y$ is a positive real number $\}$ where $k$ is a positive integer and $p_{\imath}$ is a fixed rational number for $1 \leqq i<k$ if $k>1$.

Consider the subset $M$ of the upper plane such that $M=\{(x, y) \mid x>0$ and $y \geqq 0\}$. To each line $L$ as above associate a unique copy $M_{L}$ of $M$ such that $L$ is identified with the nonnegative $x$-axis in $M_{L}$.

Now for each line $L$, consider $M_{L}$ as a subset of the plane with the usual topology. Suppose $n$ is a positive integer and $p$ is a point of $M_{L}$. (i) If $p$ is a point in $M_{L}$ not in $L$, let $g_{n}(p)$ denote the common part of $M_{L}$ and 
the interior of a circle about $p$ with radius equal to the lesser of $1 / n$ and the ordinate of $p$. (ii) If $p=\left(p_{1}, p_{2}, \cdots, p_{k-1}, y, 0, \cdots\right)$ is a point of $L$ such that $y$ is irrational, let $u_{n}(p)$ denote the common part of $M_{L}$ and the interior of a circle of radius $1 / n$ lying wholly above the $x$-axis and tangent to the $x$-axis at $p$ together with the point $p$.

Note that for each point $p=\left(p_{1}, p_{2}, \cdots, p_{k}, 0, \cdots\right)$ in $S$ such that $p_{k}$ is irrational, $u_{n}(p)$ is uniquely defined for each positive integer $n$. Also, since there are only countably many lines in $S,\left\{M_{L} \mid L\right.$ a line in $\left.S\right\}$ is countable.

4. Points of $X$. Let $X$ be the set to which $p$ belongs if and only if $p$ is a point of $S$ or $p$ is a point of $M_{L}$ for some line $L$ in $S$.

5. Regions of $X$. Suppose $n$ is a positive integer and $p$ is a point of $X$. (i) If $p=\left(p_{1}, p_{2}, \cdots, p_{k}, 0, \cdots\right)$ is a point of $S$ such that $p_{k}$ is irrational, let

$$
h_{n}(p)=g_{n}(p) \cup\left(\bigcup\left\{u_{n}(q) \mid q \in g_{n}(p)\right\}\right) .
$$

(ii) If $p=\left(p_{1}, p_{2}, \cdots, p_{k}, 0, \cdots\right)$ is a point of $S$ such that $p_{k}$ is rational, let

$$
\begin{array}{r}
h_{n}(p)=g_{n}(p) \cup\left(\bigcup \left\{h_{n}(q) \mid q \in g_{n}(p) \text { and } q=\left(q_{1}, q_{2}, \cdots, q_{m}, 0, \cdots\right)\right.\right. \\
\text { in } \left.\left.S \text { where } q_{m} \text { is irrational }\right\}\right) .
\end{array}
$$

(iii) If $p$ is a point of $M_{L}$ for some line $L$ and $p$ is not in $L$, let $h_{n}(p)=g_{n}(p)$.

6. Properties of $X$. For each positive integer $i$, let $H_{i}^{\prime}=\left\{h_{i}(p) \mid p \in S\right\}$. For each positive integer $j$, let $H_{j}=\bigcup_{i=j}^{\infty} H_{i}^{\prime}$. It follows that $X$ is a Moore space and $H_{1}, H_{2}, \cdots$ is a development for $X$. Also, since each open set in $X$ contains a subset which is open (with respect to the topology of the plane) in $M_{L}$ for some line $L$ in $S$, then $X$ is separable. Finally, the space $S$ is a subspace of $X$, thus $X$ is not completable.

\section{REFERENCES}

1. S. Armentrout, Completing Moore spaces, Topology Conference (Arizona State Univ., Tempe, Ariz., 1967), Arizona State Univ., Tempe, Ariz., 1968, pp. 22-35. MR 39 \#2123.

2. B. Fitzpatrick, Jr., On dense subspaces of Moore spaces, Proc. Amer. Math. Soc. 16 (1965), 1324-1328. MR 32 \#1663.

3. G. D. Creede, Embedding of complete Moore spaces, Proc. Amer. Math. Soc. 28 (1971), 609-612. MR 43 \#1119.

4. R. L. Moore, A set of axioms for plane analysis situs, Fund. Math. 25 (1935), 13-28.

5. - - - Foundations of point set theory, rev. ed., Amer. Math. Soc. Colloq. Publ., vol. 13, Amer. Math. Soc., Providence, R.I., 1962. MR 27 \#709. 
6. J. W. Ott, Subsets of separable spaces, Doctoral Dissertation, Auburn University, Auburn, Ala., 1969.

7. C. Pixley and P. Roy, Uncompletable Moore spaces, Proc. Auburn Topology Conference, Auburn, Ala., 1969, pp. 75-85.

8. G. M. Reed, Concerning normality, metrizability and the Souslin property in subspaces of Moore spaces, General Topology and Appl. 1 (1971), 223-246.

9. J. H. Roberts, A property related to completeness, Bull. Amer. Math. Soc. 38 (1932), 835-838.

10. M. E. Estill (Rudin), Concerning abstract spaces, Duke Math. J. 17 (1950), 317-327. MR 13, 148.

11. - Separation in non-separable spaces, Duke Math. J. 18 (1951), 623-629. MR 13, 148.

12. J. N. Younglove, Concerning dense metric subspaces of non-metric spaces, Fund. Math. 48 (1959), 15-25. MR 22 \#1878.

Department of Mathematics, Auburn University, Auburn, Alabama 36830

Department of Mathematics, Ohio University, Athens, Ohio 45701 (Current address) 